\title{
Fast Exposure Fusion Based on Histograms Segmentation
}

\author{
Mohammed Elamine Moumene ${ }^{1}$, Rachid Nourine ${ }^{1}$, and Djemel Ziou ${ }^{2}$ \\ 1 Université d'Oran, Laboratoire LITIO, BP 1524, \\ El-M'Naouer, 31000, Oran, Algeria \\ Elamine.Moumene@gmail.com, Rachid.Nourine@univ-oran.dz \\ 2 Département d'Informatique, Université de Sherbrooke, QC, J1K 2R1, Canada \\ Djemel.Ziou@usherbrooke.ca
}

\begin{abstract}
Usual cameras can gather only a small interval of intensities found in high dynamic range scenes. This fact leads to loss of details in acquired images and apparition of under or overexposed pixels. A popular approach to deal with this problem is to take several images differently exposed and fuse them into one single image. The exposure fusion is mostly performed as a weighted average between corresponding pixels. Weighting all pixels of the exposure bracketing slows the fusion process and makes realtime acquisition difficult. In this paper we present a fast exposure fusion method based on histograms segmentation. The segmentation phase reduces considerably the computations while preserving competitive fusion quality. We present also an automatic way to take enhanced exposures for fusion, using the segmented regions. Subjective and objective comparisons are conducted to prove the effectiveness of our method.
\end{abstract}

Keywords: High dynamic range, exposure fusion, weighting function.

\section{Introduction}

Only a small part of the large intensities interval found in high dynamic range scenes can be captured with the 8 bit per pixel image sensor. Two approaches are distinguished in the literature treating how to take good images facing this situation. The first one deals with camera response function estimation to create radiance map [5]. Tone mapping algorithm is required to transform the radiance map to a displayable image. The second approach proceeds directly with fusing a sequence of differently exposed images to obtain enhanced low dynamic range image [11/10/14. The second approach is preferred because of its simplicity, it provide time saving, even though it produces comparable results to the first one. The dominant technique for exposure fusion is to perform a weighted average between corresponding pixels, where weights are attributed using quality measures 11]10 14. Most used quality measures are contrast, saturation and well exposedness introduced by Mertens et al. [11. Estimating those metrics for every pixel slow the fusion process and make realtime processing difficult. Mertens exposure

A. Elmoataz et al. (Eds.): ICISP 2014, LNCS 8509, pp. 367-374, 2014.

(C) Springer International Publishing Switzerland 2014 
fusion was dedicated at first to capture still images, using fixed cameras. Movements of objects between frames generate ghosting artifacts in the fused image. A large number of de-ghosting methods appears to deal with dynamic scenes. Zhang et al. [14 improve Mertens exposure fusion by introducing a novel criterion called temporal consistency which is based on gradient direction. Starting from one exposure as a reference view, they suppose that the gradient direction of well exposed pixels is invariant in the other exposures. Chapiro et al. 44 also improve Mertens weighting technique with a fourth numerical parameter called ghosting. It defines the likelihood of presence of object movement on the scene at the current pixel using filters. Those de-ghosting methods still have difficulties with quick movements and consume additional processing time [8] which makes realtime fusion harder.

In this paper we present a fast exposure fusion method dedicated to real time acquisition. When exposures acquisition and fusion are fast enough, motion between frames will be negligible and there will be no need for de-ghosting methods. Our main contribution consists of using histograms segmentation to detect shadows, highlights and intermediate regions of the scene. We use this segmentation phase to avoid lot of computations and to eliminate influence of saturated or darkened pixels on the fused image quality. We demonstrate also how the segmented regions are used with auto-exposure control of the camera to provide enhanced exposure bracketing for fusion.

The rest of this paper is organized as follow: the section two introduces the exposures segmentation method, explains how it is used to fuse exposures fast and how the segmented regions are used to provide enhanced exposure bracketing for fusion. Section three shows obtained results and comparisons with two relevant works. Finally, the conclusion is presented in section four.

\section{Fast Exposure Fusion Based on Histograms Segmentation}

Usual exposure fusion methods calculate weights for every pixel from the inputs using quality measures, and then proceed to fusion with a weighted average 111014. In our work, we first detect shadows, highlights and intermediate regions. The fused image is constructed directly with segmented shadows and highlights without use of weighting or summation operations. In addition to reduce considerably the amount of operations, this technique avoids influence of saturated or dark pixels on the fused image quality as explained later in the experimentations section (section 3 ). The majority of exposure fusion works which aim real time image fusion consider that fusing two exposures is adequate 489. Using only two exposures saves processing time and recovers most of the scene details. We aim for realtime exposure fusion dedicated for video capture. This is why we consider also only two exposures: an overexposed image $O$ and another image $U$ which is underexposed. The proposed exposures segmentation phase is based on histograms noted $H_{k}(L)$, which represent the number of pixels found in image $k \in\{U, O\}$ having the $L^{t h}$ intensity value. Shadows are pixels 

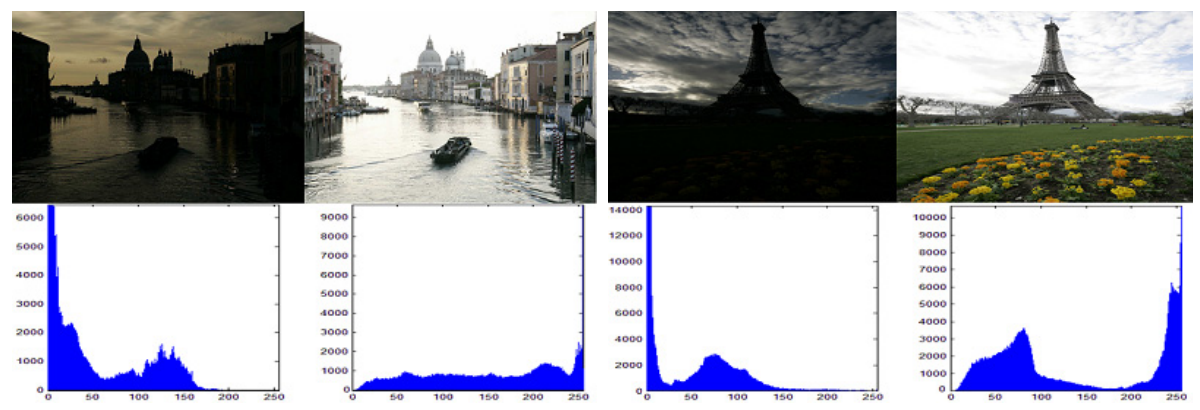

Fig. 1. Exposures and their intensity histograms ("venise" and "Eiffeltour" inputs, image courtesy of Jacques Joffre [1.)

having small intensities in underexposed image. They form a high histogram mode positioned at the extreme left of $H_{U}$. Highlights are pixels having high intensities in the overexposed image histogram. This is reflected by apparition of high mode at the extreme right of $H_{O}$. Those assumptions are realistic as it is demonstrated in Fig. 1 showing exposures and their histograms. Shadows and highlights are then looked as two modes that we represent by Gaussian functions. The segmentation phase is carried out as two Gaussians parameters estimation, which are maximums and inflection points $\left(M_{U}, T_{U}, M_{O}, T_{O}\right)$. We pose $M_{U}$ and $M_{O}$ on the maximums found inside the interval $[0,255]$ of the corresponding histograms $H_{U}$ and $H_{O}$. The inflection points $T_{U}$ and $T_{O}$ are estimated using the first and second derivatives of the smoothed histograms:

$$
\begin{aligned}
& T_{U}=\min \left\{i \in[0,255] \text { where } H_{U}^{\prime \prime}(i) \times H_{U}^{\prime \prime}(i-1)<=0 \text { and } H_{U}^{\prime}(i)<0\right\} \\
& T_{O}=\max \left\{i \in[0,255] \text { where } H_{O}^{\prime \prime}(i) \times H_{O}^{\prime \prime}(i-1)<=0 \text { and } H_{O}^{\prime}(i)>0\right\}
\end{aligned}
$$

Once the inflection points are estimated, we detect well exposed pixels of shadows (SH) and highlights (HI) with rules (3 and 4). Intermediate regions (IR) are detected with rule (5). Fig. 2 shows an example of exposures segmentation using the detected inflection points.

$$
\begin{gathered}
S H=\left\{p_{x, y} \in O \text { where } U_{x, y}<=T_{U}\right\} \\
H I=\left\{p_{x, y} \in U \text { where } O_{x, y}>=T_{O}\right\} \\
I R=\left\{p_{x, y} \in\{U \cup O\} \text { where } U_{x, y}>T_{U} \text { and } O_{x, y}<T_{O}\right\}
\end{gathered}
$$

After the segmentation phase, the fused image $R$ is constructed directly with ( $\mathrm{SH}$ and $\mathrm{HI}$ ) pixels because their corresponding pixels in the other exposures are 


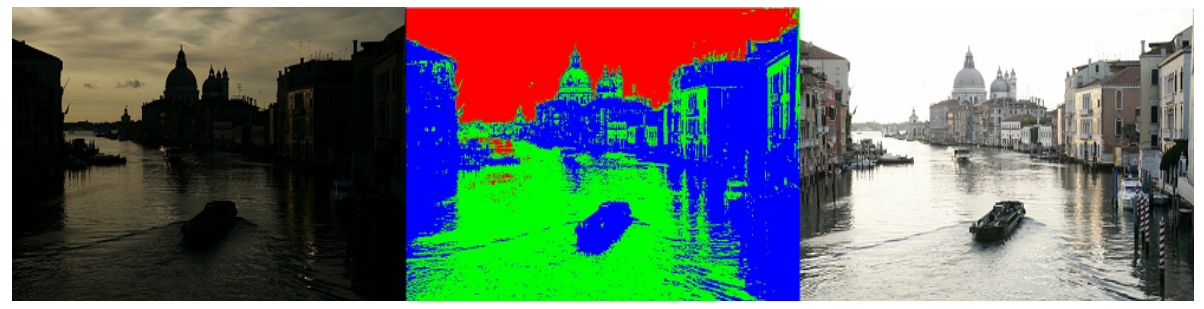

Fig. 2. Exposures segmentation using the detected inflection points. Red color: Highlights, Blue color: Shadows, Green color: Intermediate regions ("venise" inputs, image courtesy of Jacques Joffre [1].)

darkened or saturated. Only intermediate regions need weighted fusion because they are common data between the two exposures. For that, we assume that the more bright are the pixels in the underexposed image, the more they are relevant. In the overexposed image, the more bright are the pixels, the less they are pertinent. This weighting approach is formalized by two simple functions (6 and 7) based on the estimated thresholds. The final values of intermediate pixels are calculated using (8).

$$
\begin{gathered}
W_{1}\left(U_{x, y}\right)=U_{x, y}-T_{U} . \\
W_{2}\left(O_{x, y}\right)=T_{O}-O_{x, y} \\
R_{x, y}=\left(U_{x, y} \times W_{1}\left(U_{x, y}\right)+O_{x, y} \times W_{2}\left(O_{x, y}\right)\right) /\left(W_{1}\left(U_{x, y}\right)+W_{2}\left(O_{x, y}\right)\right)
\end{gathered}
$$

Fig. 3a shows an unsatisfactory image fusion. This is a known issue in exposure fusion caused by the fact that combined images contain different absolute intensities due to their different exposure times [1]. Mertens overcomes this problem with a multi-resolution blending [11, which is time consuming. Another solution is to process inputs with histogram equalization as done in [10, but it produces strong deformation of intensities resulting sometimes to poor image quality (see Fig. 3c). To obtain a consistent fusion with minimum of processing time, we use two linear functions (9 and 10) that bring closer inputs intensities (reduce luminance of $O$ and increase it in $U)$. We set $(d=2.3)$. Fig. 3b shows the fused image after adjusting inputs with (9 and 10).

$$
\begin{gathered}
u(x, y)=U_{x, y}+\left(255-U_{x, y}\right) / d \\
o(x, y)=O_{x, y}-O_{x, y} / d .
\end{gathered}
$$

How to take the two exposures used for fusion is an important part of the problem. The usual manner to take those inputs is based on fixed parameters or 


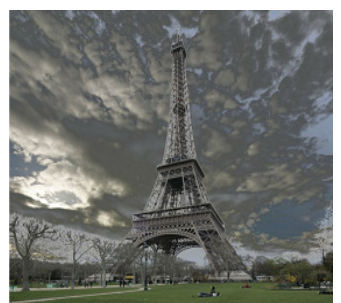

(a)

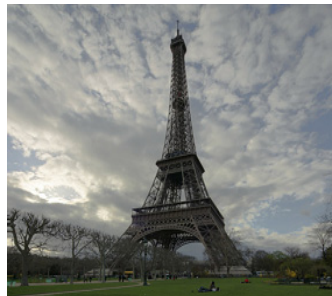

(b)

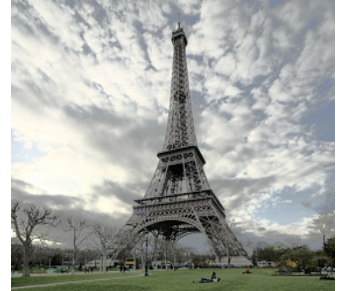

(c)

Fig. 3. Inputs luminance adjustment then fusion. (a) Without any adjustement, (b) With the proposed luminance adjustement (c), With histogram equalization ("Eiffeltour" inputs, image courtesy of Jacques Joffre [1].)

thresholds, which lead often to loss of details in shadows or highlights. Gelfand et al. 6] suppose that $O$ is captured when the number of dark pixels (luminosity< 16 ) is inferior to $10 \%$ and that $U$ is taken when less than $10 \%$ of pixels have intensity greater than 239. Bilcu et al. 3], Marius et al. 13. propose also exposure bracketing algorithms based on fixed thresholds. A more sophisticated technique to get the exposures is to adjust exposure parameters according to quality of regions of interest (ROI). Kao 9 adjusts exposures according to the detected human skin color. In our work, the first exposure bracketing is captured using fixed parameters as done in [6]. Then $U$ and $O$ are segmented using the explained technique above. The detected regions ( $\mathrm{SH}$ and $\mathrm{HI}$ ) are processed with autoexposure control of the camera in turn to deliver respectively overexposed image and underexposed one. We used the frankencamera architecture of the Nokia N900 [2] for implementation. The implanted Auto-exposure control of Marius Tico [2] adjusts exposure time and gain according to image histogram, in such way that a percentage of pixels $P=0.9$ hit a brightness value $B=0.4$. We adapt this algorithm so that it accounts only SH and HI in turn. Fig. 4a and Fig. 4b show the obtained exposures. Fig. 4c and Fig. 4d shows shadows and highlights acquisition using the Fcam application of the Nokia N900. We can see that our technique brings more details in some regions.

\section{Results and Comparisons}

To prove the effectiveness of the proposed exposure fusion method, we compare it with Mertens algorithm [1] and Photomatix exposure fusion [1]. Fig. 5 shows outputs of the three methods. Subjectively, we can see that our method brings more details in some regions comparing to Mertens algorithm (the boat in "Venise" and the tour edges in "Eiffeltour"). This is because segmented pixels of shadows and highlights form directly our fused image, without being summed with their corresponding pixels which are saturated or dimmed. Mertens algorithm use a Gaussian weighting function which assigns same weights to those 


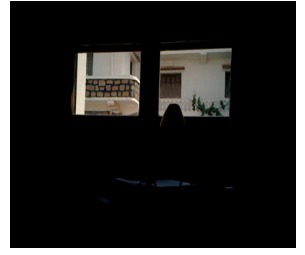

(a)

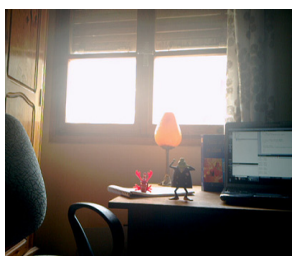

(b)

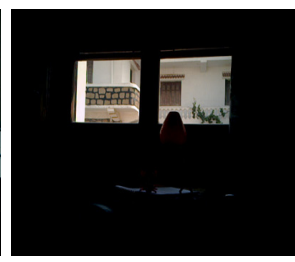

(c)

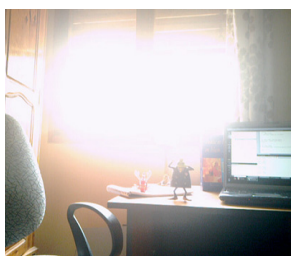

(d)

Fig. 4. Automatic exposure bracketing. $(\mathrm{a}, \mathrm{b})$ : shadows and highlights from our acquisition. (c,d) : Fcam application [2] for shadows and highlights acquisition. (Exposure time, gain): (a) $(1666,1),(\mathrm{b})(40000,1.58),(\mathrm{c})(2397,1),(\mathrm{d})(40000,6.68)$.

regions in both of exposures. Visual comparison of our method with Photomatix shows that the two methods are close to each other. We used three quality measures to perform objective comparisons. The first one is mutual information 7], adapted for the image fusion performance evaluation. Furthermore, we calculated the color difference using CIEDE2000 [12] and contrast difference (gradient magnitude). Ground truth images are constructed manually with best parts of inputs and without deformation of pixels values. Results presented in Table 1 shows that our method provides generally best performance in term of mutual information, CIEDE2000 and contrast difference.

Table 1. Objective Comparisons: Contrast (Gradient Magnitude Difference), Color (Ciede2000 [12]) and Mutual Information 7] (Image courtesy of Jacques Joffre [1])

\begin{tabular}{|c|c|c|c|c|c|}
\hline Metric & Methods & Venise & Eiffel & Lighthouse & Landscape \\
\hline \multirow{3}{*}{ 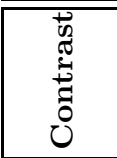 } & Mertens & 1,15 & 0.85 & 0.32 & 1.53 \\
\hline & Our Method & 1.05 & 0.71 & 0.27 & 1.34 \\
\hline & Photomatix & 1.13 & 0.72 & 0.30 & 1.40 \\
\hline \multirow{3}{*}{$\frac{\partial}{0}$} & Mertens & 1.56 & 2.89 & 1.17 & 1.26 \\
\hline & Our Method & 0.99 & 1.99 & 1.10 & 1.18 \\
\hline & Photomatix & 0.94 & 1.79 & 1.63 & 1.57 \\
\hline \multirow{3}{*}{$\sum$} & Mertens & 2.934 & 3.427 & 2.779 & 4.551 \\
\hline & Our Method & 6.699 & 7.463 & 5.570 & 7.734 \\
\hline & Photomatix & 5.200 & 6.292 & 6.306 & 6.880 \\
\hline
\end{tabular}

In the Table 2 we see that detected shadows and highlight represent a significant part of the exposure bracketing. Those regions are directly picked to form the fused image without any weights estimation. This is why our method is fast (see Table 2). C++ version of the fusion application, running on 2.0GHZ CPU and $3 \mathrm{GO}$ of memory, achieved colored images with frame rate of 29 fps having resolution of $640 \times 480$. 

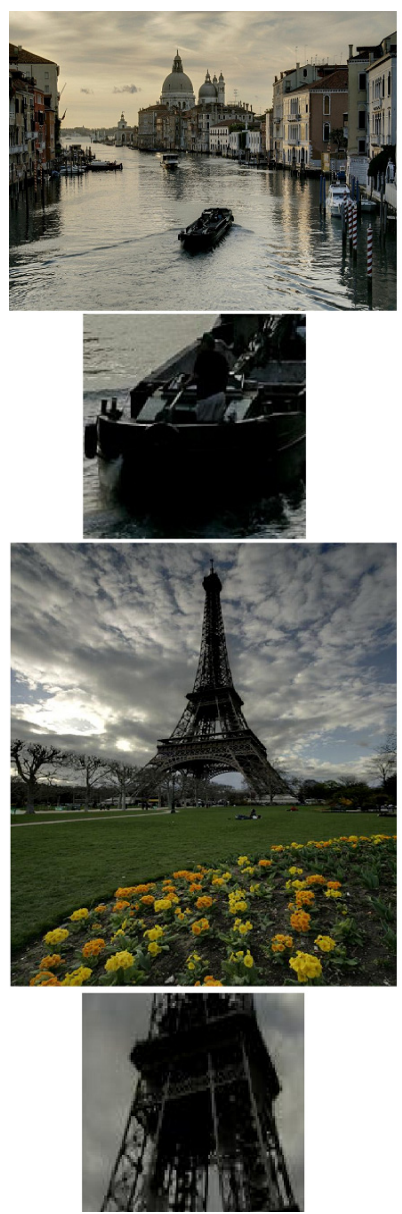

(a)
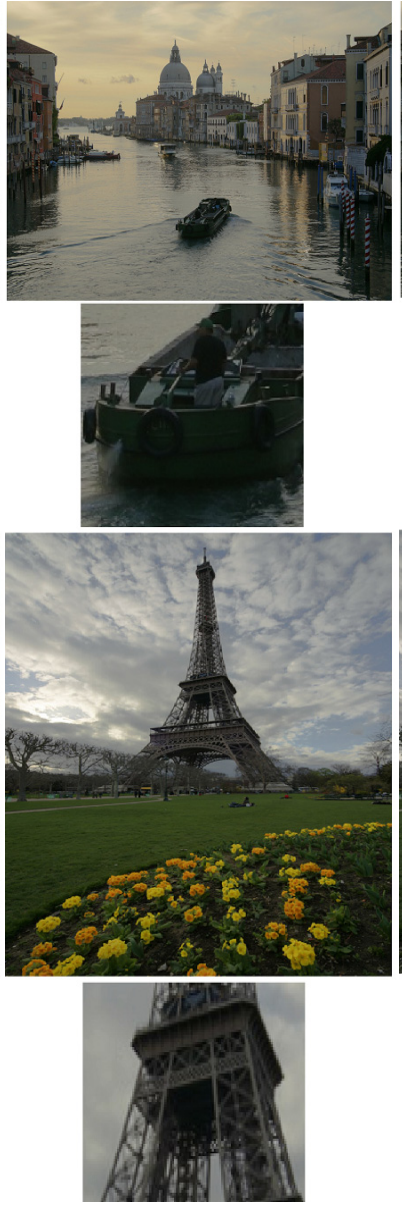

(b)
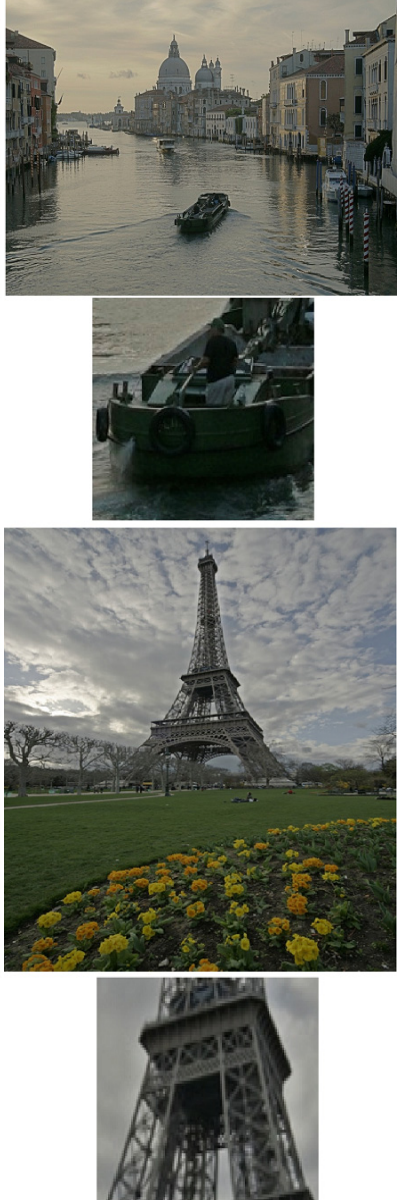

(c)

Fig. 5. Outputs of the three methods. (a): Mertens [11. (b): Our Method. (c): Photomatix. (Image courtesy of Jacques Joffre [1])

Table 2. Processing Time of Exposure Fusion methods in Seconds (Matlab Implementations) and percentage of segmented shadows and highlights pixels

\begin{tabular}{|c|c|c|c|c|}
\cline { 2 - 5 } \multicolumn{1}{c|}{} & Venise & Eiffel tour & Lighthouse & Landscape \\
\hline Resolution & $640 \times 480$ & $530 \times 795$ & $1024 \times 682$ & $1800 \times 1198$ \\
\hline SH+HI $(\%)$ & 63.99 & 69.51 & 60.28 & 46.53 \\
\hline Mertens & 2.1 & 2.5 & 3.4 & 10.4 \\
\hline Our Method & 0.3 & 0.5 & 1.1 & 2.4 \\
\hline
\end{tabular}




\section{Conclusion}

In this paper we presented an exposure fusion method which meets realtime requirements while preserving competitive image quality to some relevant works. Achieving at least 29 colored images per second, our method delivers enhanced images facing high dynamic range scenes. The proposed histograms segmentation can be the starting point of any exposure fusion algorithm to save processing time and eliminate influence of saturated or dark pixels on the fused images quality. Using the segmented regions with auto-exposure control of camera provided an automatic way to acquire enhanced exposure bracketing for fusion.

\section{References}

1. hdrsoft, http://www.hdrsoft.com

2. Adams, A., Talvala, E.V., Park, S.H., Jacobs, D.E., Ajdin, B., Gelfand, N., Dolson, J., Vaquero, D., Baek, J., Tico, M., Lensch, H.P.A., Matusik, W., Pulli, K., Horowitz, M., Levoy, M.: The frankencamera: An experimental platform for computational photography. In: SIGGRAPH (2010)

3. Bilcu, R.C., Burian, A., Knuutila, A., Vehvilainen, M.: High dynamic range imaging on mobile devices. In: 15th IEEE International Conference on Electronics, Circuits and Systems, ICECS 2008, pp. 1312-1315 (August 2008)

4. Chapiro, A., Cicconet, M., Velho, L.: Filter based deghosting for exposure fusion video. In: SIGGRAPH (2011)

5. Debevec, P.E., Malik, J.: Recovering high dynamic range radiance maps from photographs. In: The 24th Annual Conference on Computer Graphics and Interactive Techniques. pp. 369-378 (1997)

6. Gelfand, N., Adams, A., Park, S.H., Pulli, K.: Multi-exposure imaging on mobile devices. In: MM 2010 Proceedings of the International Conference on Multimedia, pp. 823-826 (2010)

7. Guihong, D.Z., Pingfan, Y.: Information measure for performance of image fusion. Electronics Letters 38(7), 313-315 (2002)

8. Kang, S.B., Uyttendaele, M., Winder, S., Szeliski, R.: High dynamic range video. ACM Transactions on Graphics 22(3), 319-325 (2003)

9. Kao, W.C.: Real-time image fusion and adaptive exposure control for smart surveillance systems. Electronics Letters 43(18), 975-976 (2007)

10. Li, S., Kang, X.: Fast multi-exposure image fusion with median filter and recursive filter. IEEE Transactions on Consumer Electronics 58(2), 626-632 (2012)

11. Mertens, T., Kautz, J., Reeth, F.V.: Exposure fusion: A simple and practical alternative to high dynamic range photography. Comput. Graph. Forum 28, 161-171 (2009)

12. Sharma, G., Wu, W., Dalal, E.N.: The ciede2000 color-difference formula: Implementation notes, supplementary test data, and mathematical observations. Color Research \& Application 30(1), 21-30 (2005)

13. Tico, M., Gelfand, N., Pulli, K.: Motion blur free exposure fusion. In: IEEE International Conference on Image Processing (ICIP), pp. 26-29 (September 2010)

14. Zhang, W., Cham, W.K.: Reference-guided exposure fusion in dynamic scenes. Journal of Visual Communication and Image Representation 23(3), 476-475 (2012) 